DEMENTIA

\section{Anticholinergic medications might increase the risk of AD}

According to a new study, use of medications that inhibit cholinergic activity_including some first-generation antihistamines and tricyclic antidepressants-could increase patients' risk of developing dementia. The finding could have serious implications, as some estimates suggest that as many as $37 \%$ of older adults use medications with anticholinergic effects.

"Strong anticholinergic medications can cause acute impairment in aspects of cognition," explains Shelly Gray, lead author of the new report. "However, the general thinking was that any effect on cognition was reversible once the medication was discontinued." A previous study had suggested a link between dementia and prior use of anticholinergic drugs, but questions remained over what level of exposure-in terms of dose and duration-conferred a substantial risk.

Gray and colleagues prospectively followed a cohort of 3,434 people aged over 65 years who were enrolled in a Group Health Cooperative (WA, USA) health-care plan. At study entry, and every 2 years thereafter, participants' cognitive function was assessed using the Cognitive Abilities Screening Instrument. Every member of the study cohort was cognitively normal at the beginning of the study, and participants who showed signs of impairment during the follow-up period received a standardized dementia screening protocol.

The investigators extracted data on medication use from Group Health's dispensary database, looking for drugs that are generally considered to inhibit cholinergic activity as a principal or auxiliary effect. Because the potency of each drug's effects on the cholinergic system varied, the investigators converted participants' regimens into a total standardized daily dose. The cohort was then split into five groups according to overall anticholinergic burden.

After a mean follow-up period of 7.3 years, nearly 800 participants (23\%) had developed dementia, the majority of whom (80\%) were suspected to have Alzheimer disease (AD). The risk of developing dementia was significantly higher in the group of patients with the highest anticholinergic burden (HRs 1.54 for dementia and 1.63 for probable AD) compared with the people who did not use this type of medication. These effects were independent of previously known risk factors for $\mathrm{AD}$, including age, $A P O E$ genotype and recent history of a depressive episode.

"The study reveals that people with the highest anticholinergic useequivalent to, for example, at least $10 \mathrm{mg}$ /day of doxepin, $4 \mathrm{mg} /$ day of chlorpheniramine or $5 \mathrm{mg}$ /day of oxybutynin for more than 3 yearswould be at greater risk for developing dementia," concludes Gray. "Older adults should be advised to inform their health-care providers of all over-the-counter medication use so that providers can account for all anticholinergic medications and advise on how to reduce use if possible."

Gray reports that a subset of the study cohort has consented to postmortem analysis. This follow-up investigation should help uncover the mechanism by which use of anticholingeric medications confers a greater risk of dementia, and might also provide valuable insight into the pathogenesis of AD.

Alex Chase

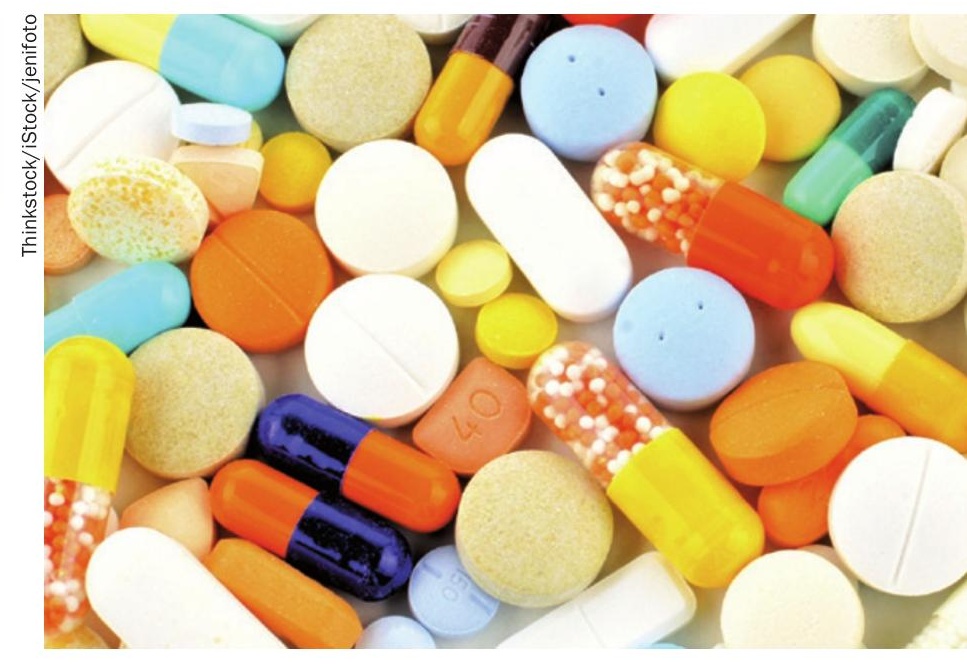

